08,09

\title{
Кинетика импульсной катодолюминесценции кристаллов ортобората лития-гадолиния, легированного примесью церия
}

\author{
(C) М.С. Киселева ${ }^{1}$, И.Н. Огородников ${ }^{1}$, В.Ю. Яковлев ${ }^{2}$ \\ ${ }^{1}$ Уральский федеральный университет, \\ Екатеринбург, Россия \\ ${ }^{2}$ Национальный исследовательский Томский политехнический университет, \\ Томск, Россия \\ E-mail: Kiseleva.Marija@gmail.com
}

\begin{abstract}
С применением времяразрешенных методов импульсной абсорбционной спектроскопии и импульсной катодолюминесценции при возбуждении электронным пучком наносекундной длительности выполнено исследование кинетики релаксации электронных возбуждений в монокристаллических и кристалловолоконных образцах $\mathrm{Li}_{6} \mathrm{GdB}_{3} \mathrm{O}_{9}$ при $293 \mathrm{~K}$. На основании результатов численного моделирования рекомбинационных процессов предложен механизм возбуждения импульсной катодолюминесценции.
\end{abstract}

DOI: $10.21883 /$ FTT.2019.05.47585.15F

\section{1. Введение}

Оптические кристаллы двойных боратов лития с общей формулой $\mathrm{Li}_{6} M e \mathrm{~B}_{3} \mathrm{O}_{9}(M e=\mathrm{Gd}, \mathrm{Eu}, \mathrm{Y})$ имеют потенциально широкую область практических применений. Во-первых, они обладают широкой полосой оптической прозрачности, простирающейся в ультрафиолетовую (УФ) область спектра. Так, коротковолновая граница оптического пропускания $\mathrm{Li}_{6} \mathrm{GdB}_{3} \mathrm{O}_{9}$ (LGBO) определяется пороговой энергией оптических переходов в матричных ионах $\mathrm{Gd}^{3+}$ (около $4.0 \mathrm{eV}$ ), тогда как пороговая энергия оптических переходов в анионной подрешетке составляет $E_{g}=9.3 \mathrm{eV}$ [1]. Значительную роль в спектре низкоэнергетических электронных переходов LGBO играют переходы с переносом заряда $\mathrm{O}-\mathrm{Gd}$, максимальное проявление которых наблюдается в области 7-8 eV [2]. Во-вторых, эти кристаллы обладают высокой изоморфной емкостью по отношению к трехвалентным примесным ионам, которые замещают матричные ионы $\mathrm{Gd}^{3+}$. Миграция электронных возбуждений в кристалле LGBO происходит по цепочкам ионов $\mathrm{Gd}^{3+}$, что обеспечивает эффективный перенос энергии электронных возбуждений от матрицы к трехвалентным примесным ионам. Имеют место все условия для эффективного легирования LGBO трехвалентными примесными ионами редкоземельных металлов, что является важным для практического применения LGBO в качестве излучающих сред, люминофоров или генерирующих сред в силовой коротковолновой лазерной технике и интегральной оптике. В-третьих, в формульный состав материала входят химические элементы, имеющие изотопы с экстремально высокими сечениями реакций взаимодействия с тепловыми нейтронами. Это позволило обсуждать LGBO как потенциальное рабочее вещество для сцинтилляционного детектора тепловых нейтронов $[3,4]$.

При возбуждении наносекундным пучком электронов с энергией 200-250 keV в LGBO : Се наблюдается интенсивная импульсная катодолюминесценция (ИКЛ), спектр которой сосредоточен в $d-f$ полосе люминес- ценции $\mathrm{Ce}^{3+}(2.5-3.4 \mathrm{eV})[5,6]$. Однако высокая интенсивность ИКЛ и особенности кинетики ее затухания существенно отличаются от таковых для случая прямого фотовозбуждения люминесценции $\mathrm{Ce}^{3+}$ или возбуждения этой люминесценции фотонным излучением в области межзонных переходов. Это заставляет предположить существование альтернативного механизма возбуждения ИКЛ LGBO:Cе. Действительно, предыдущие исследования выявили два важных факта. Вопервых, примесные ионы церия в LGBO : Cе могут быть в двух основных зарядовых состояниях: $\mathrm{Ce}^{3+}$ и $\mathrm{Ce}^{4+}$. Относительное содержание четырехвалентных ионов может в отдельных случаях достигать $3 / 4$ от общего содержания примеси церия $[7,8]$. Во-вторых, воздействие наносекундного электронного пучка указанной энергии приводит к формированию при комнатной температуре короткоживущих пар антиморфных дефектов литиевой подрешетки $[9,10]$. Из общих соображений очевидно, что эти две причины могут привести к появлению альтернативного механизма возбуждения ИКЛ.

Результаты изучения этого механизма в монокристаллах и кристалловолоконных образцах LGBO:Ce представлены в настоящей работе, в которой экспериментально исследовали спектры и кинетику ИКЛ и короткоживущего оптического поглощения (КОП) при возбуждении электронным пучком наносекундной длительности, а также проводили численное моделирование рекомбинационных процессов.

\section{2. Объекты и методы исследования}

В работе исследованы образцы ортобората литиягадолиния двух видов. Монокристаллы LGBO : Ce оптического качества были выращены в Институте геологии и минералогии СО РАН (г. Новосибирск). Образцы LGBO : Ce $(0.5$ at.\% Ce) были приготовлены в виде полированных плоскопараллельных пластин $7 \times 7 \times 2 \mathrm{~mm}$. Детали выращивания и спектроскопические свойства монокристаллов представлены в [11]. 
Кристалловолоконные образцы LGBO : Ce (1 ат.\% Ce, $\varnothing 800 \mu \mathrm{m}$, длина $30 \mathrm{~mm}$ ) были синтезированы И.Н. Седуновой совместно с К. Лебу (K. Lebbou), К. Педрини (Ch. Pedrini), А.В. Ищенко и Т.С. Королевой в Лионском университете (г. Лион) методом микровытягивания (Micro Pulling Down) в воздушной атмосфере [12,13]. После синтеза все кристалловолоконные образцы были подвергнуты термообработке $\left(600^{\circ} \mathrm{C}, 15 \mathrm{~h}\right.$, атмосфера аргона).

Подробное описание экспериментальной установки и особенностей методов люминесцентной и абсорбционной спектроскопии с наносекундным временным разрешением приведено в работах [14,15].

\section{3. Результаты эксперимента}

На рис. 1, а приведены времяразрешенные спектры КОП монокристалла LGBO : Ce $(T=293 \mathrm{~K})$. Все эти спектры при $E>1.2 \mathrm{eV}$ имеют вид круто нарастающей кривой, которая достигает максимума около $E=2 \mathrm{eV}$ и далее плавно спадает к $3 \mathrm{eV}$. В области $E=3.4-3.8 \mathrm{eV}$ наблюдается менее интенсивный широкий максимум.

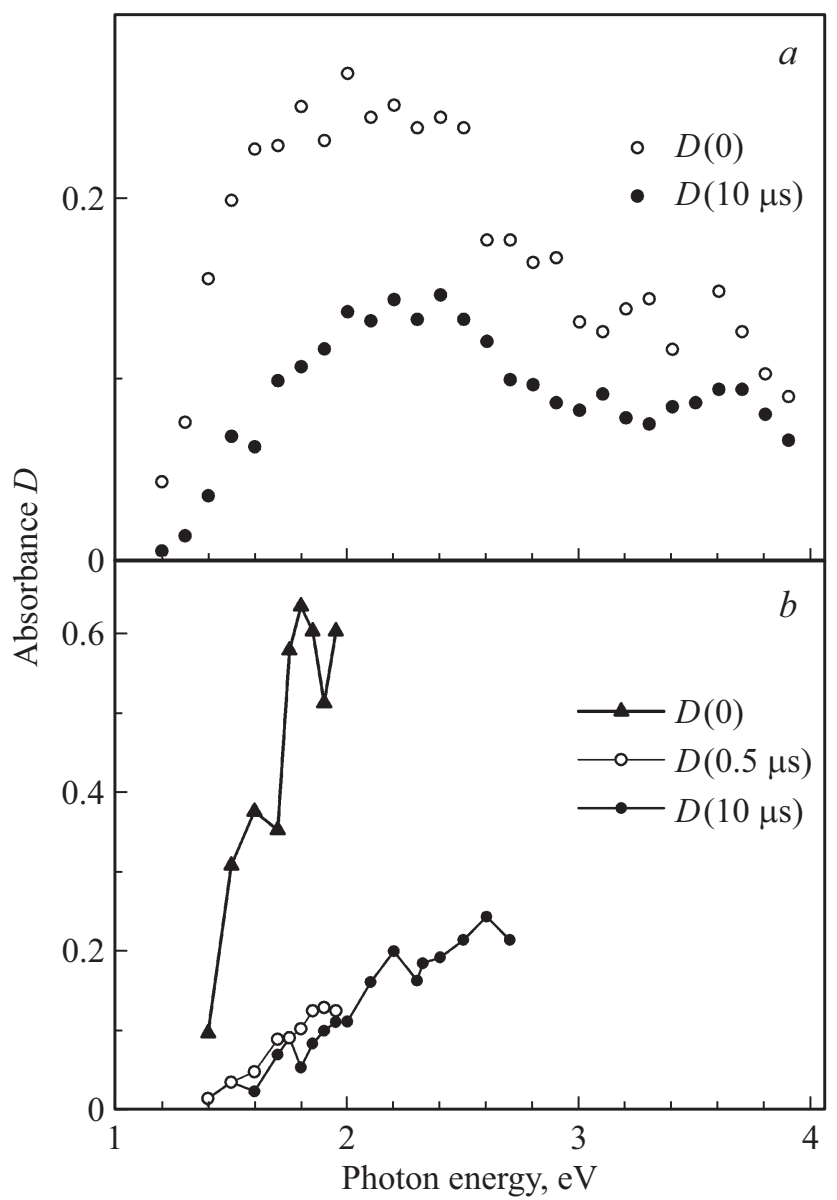

Pис. 1. Спектры КОП LGBO : Се при $T=293 \mathrm{~K}$, зарегистрированные для монокристалла $(a)$ и кристалловолокна $(b)$ сразу после окончания импульса возбуждения $(D(0))$ и с задержкой на указанное время $\Delta T(D(\Delta T))$.

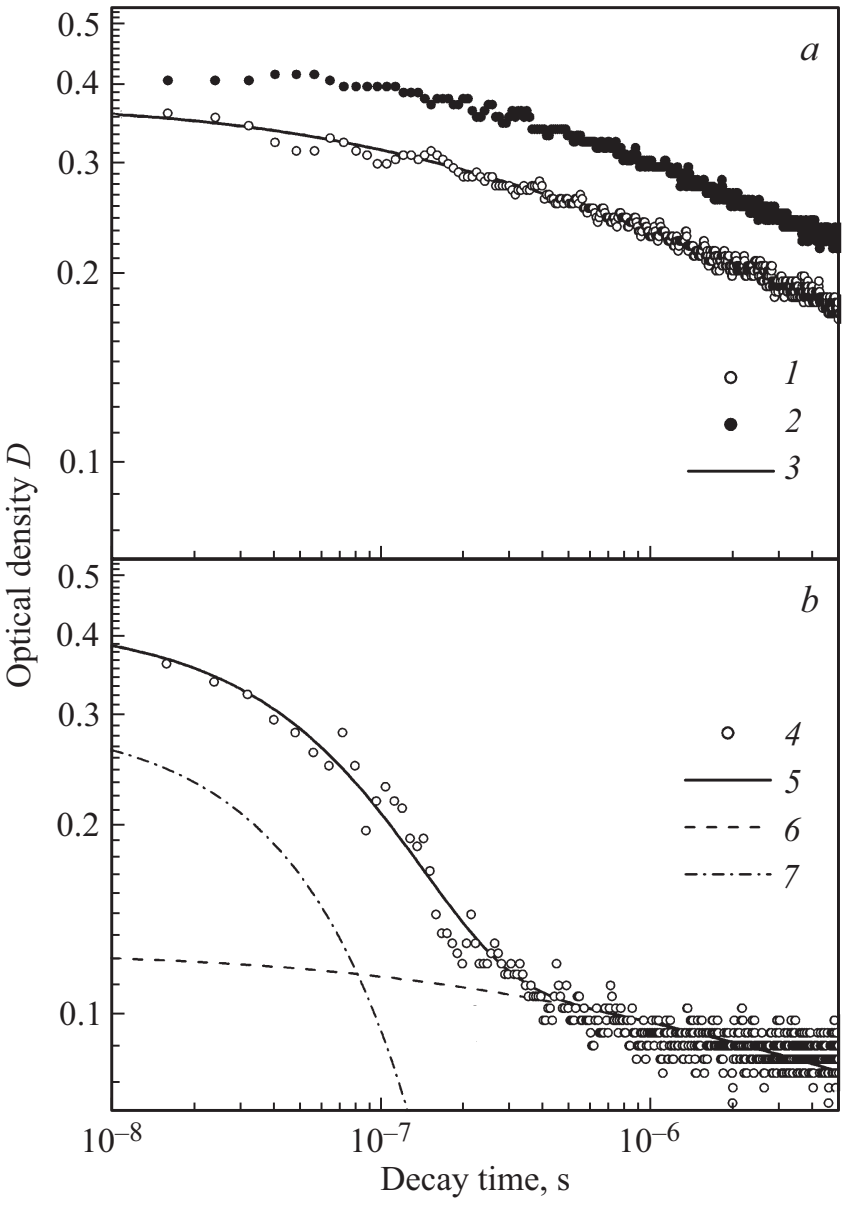

Рис. 2. Кривые кинетики затухания КОП LGBO : Ce при $T=293 \mathrm{~K}$, зарегистрированные для монокристалла $(a)$ и кристалловолокна $(b)$ в полосе при $E=1.8(1,4)$ и $2.10 \mathrm{eV}(2)$. Сплошные гладкие линии $(3,5)-$ результаты аппроксимации, штриховыми линиями показаны выделенные компоненты: туннельный (6) и термоактивационный (7).

Интенсивность сигнала $D(t)$ затухает достаточно однородно по спектру. Затухание происходит сравнительно медленно: через $10 \mu \mathrm{s}$ после возбуждения интенсивность сигнала затухает примерно в два раза.

Спектры КОП кристалловолоконных образцов (рис. $1, b)$ измерены только в ограниченных областях $E=1.4-2.0 \mathrm{eV}$ для мгновенного спектра $D(0)$ и $1.4-2.8 \mathrm{eV}$ для времяразрешенных спектров. Это обусловлено тем, что в области $2.5-3.5 \mathrm{eV}$ наблюдается ИКЛ, интенсивность которой соизмерима с интенсивностью зондирующего света при изучении КОП. Кроме того, в окрестности $E=1.8 \mathrm{eV}$ величина регистрируемой оптической плотности более чем в три раза превосходит таковую для монокристалла, что затрудняет процесс регистрации в выбранной геометрии измерений. Основное отличие спектров КОП кристалловолоконных образцов (рис. $1, b)$ - это наличие быстрого компонента затухания: в первые $500 \mathrm{~ns}$ времени затухания оптическая плотность $D(t)$ при $E=1.8 \mathrm{eV}$ уменьшается на порядок 


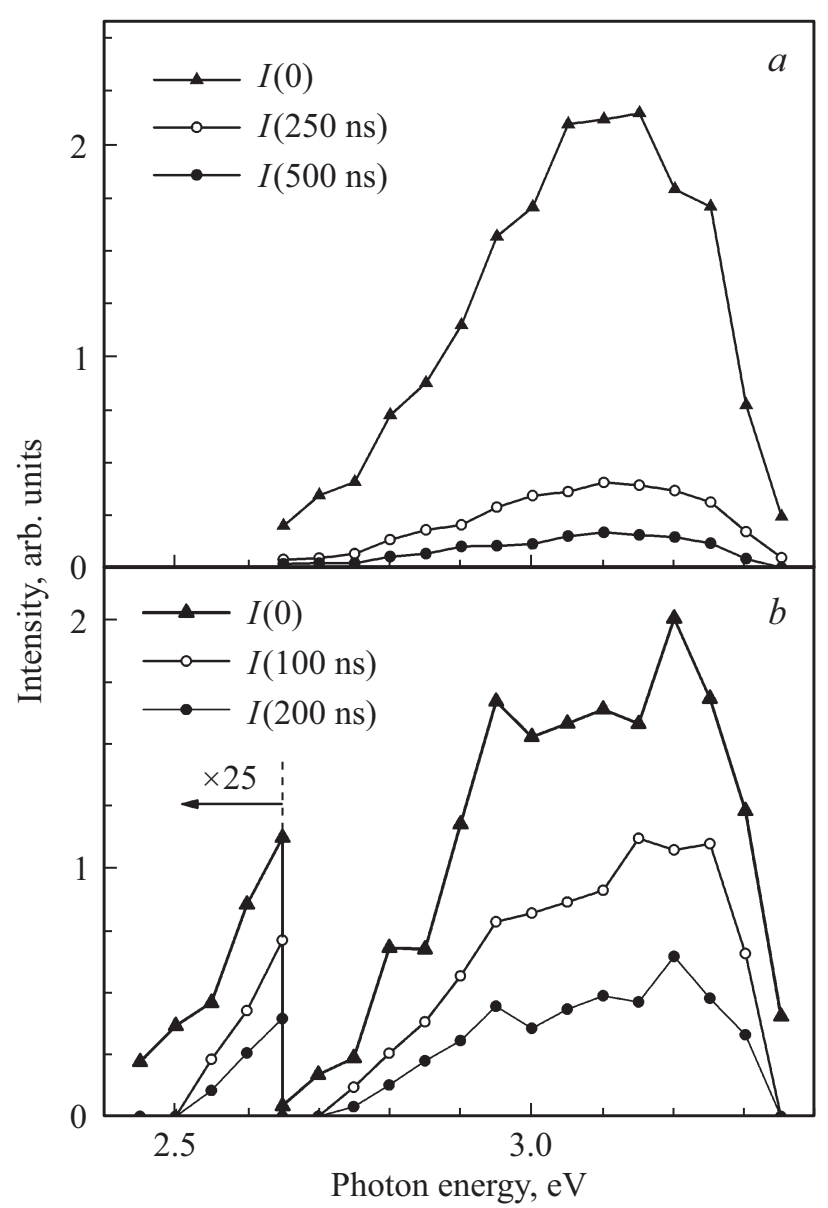

Рис. 3. Спектры ИКЛ кристалловолокна LGBO, зарегистрированные при $T=293(a)$ и $80 \mathrm{~K}(b)$ сразу после окончания импульса возбуждения $(I(0))$ и с задержкой на указанное время $\Delta T(I(\Delta T))$.

величины. При больших временах затухания скорость спада $D(t)$ соизмерима с таковой для монокристалла.

На рис. 2, а представлены две кривые кинетики затухания КОП монокристалла LGBO : Ce $(T=293 \mathrm{~K})$, зарегистрированные при $E=1.8$ и $2.1 \mathrm{eV}$. В двойных логарифмических координатах эти кинетики имеют вид плавно спадающих кривых. В области времени затухания $10 \mathrm{~ns}-5 \mu \mathrm{s}$ профили обеих кривых практически совпадают, различаясь лишь интенсивностью: величина $D(t)$ уменьшается с 0.36 до 0.18 при $E=1.8 \mathrm{eV}$, с 0.42 до 0.22 при $E=2.1 \mathrm{eV}$. Кинетика затухания КОП монокристалла была также измерена при $E=1.65 \mathrm{eV}$, но на рис. 2, $a$ она не приведена, поскольку совпадает с таковой для $E=1.8 \mathrm{eV}$.

Для измерения кинетики затухания КОП кристалловолокна были подобраны условия измерения, при которых величина сигнала $D(0)$ практически совпадала с таковой для монокристалла, рис. 2, $b$. Из рис. 2, $b$ видно, что основная доля оптической плотности затухает в интервале времени до $300 \mathrm{~ns}$. Далее имеет место медленное затухание, сопоставимое с таковым для монокристалла. Это свидетельствует о наличии в кинетике затухания
КОП кристалловолокна мощного компонента затухания с постоянной временем от нескольких десятков до сотни наносекунд.

На рис. 3 представлены времяразрешенные спектры ИКЛ кристалловолоконных образцов LGBO : Cе, зарегистрированные при $T=80$ и $293 \mathrm{~K}$. Люминесценция в области $2.5-3.4 \mathrm{eV}$ отнесена к интерконфигурационным $5 d-4 f$ переходам в примесных ионах $\mathrm{Ce}^{3+}$. Из сопоставления времяразрешенных спектров при комнатной температуре (рис. 3,a) видно, что основная доля светосуммы затухает за время короче $250 \mathrm{~ns}$. При $T=80 \mathrm{~K}$ затухание происходит сравнительно медленно.

Во всех случаях затухание происходит однородно по спектру, поэтому кривые кинетики затухания ИКЛ

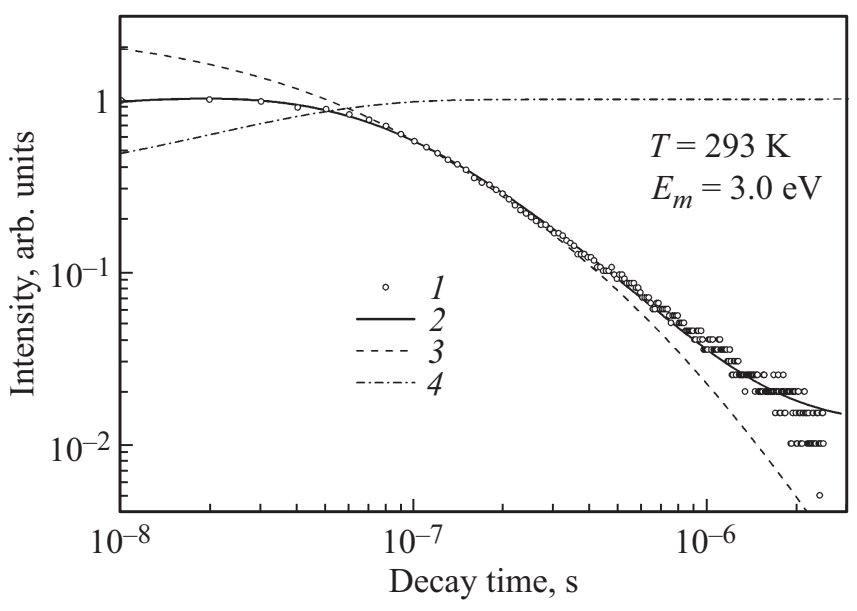

Рис. 4. Кинетика затухания ИКЛ кристалловолокна LGBO : Cе, зарегистрированная при $E_{m}=3.0 \mathrm{eV}$ и $T=293 \mathrm{~K}$ (1). Сплошная линия - результат аппроксимации (2), штриховыми линиями показаны: туннельный компонент $I_{\text {tun }}(3)$ и функция выхода $\eta(t)(4)$.

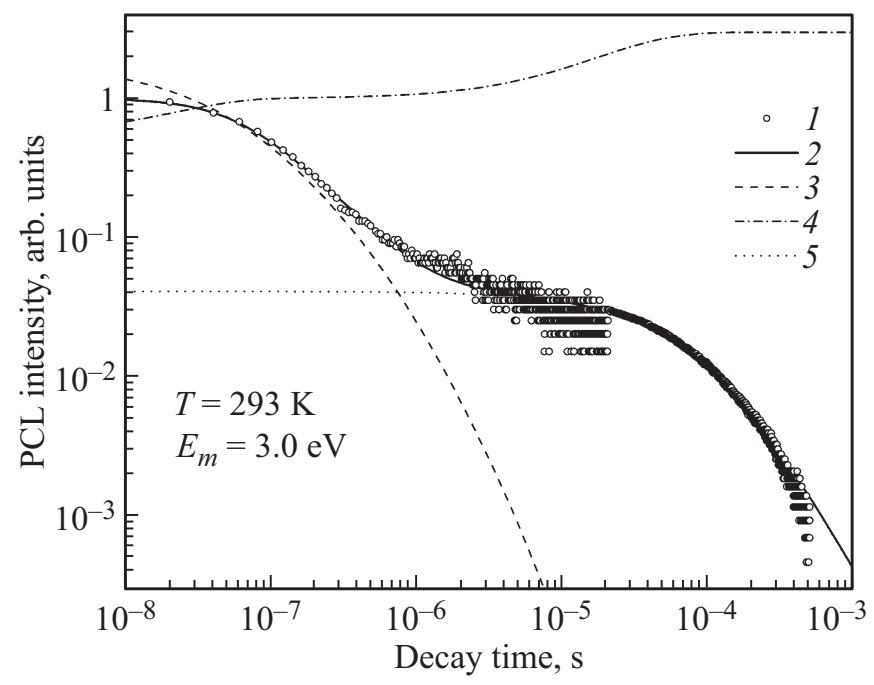

Рис. 5. Кинетика затухания ИКЛ монокристалла LGBO : Ce, зарегистрированная при $E_{m}=3.0 \mathrm{eV}$ и $T=293 \mathrm{~K}(1)$. Сплошная линия - результат аппроксимации (2), штриховыми линиями показаны: туннельный компонент $I_{\text {tun }}(3)$, функция выхода $\eta(t)(4)$ и инерционный рекомбинационный процесс (5). 
(рис. 4,5) приведены только для энергии $E_{m}=3.0 \mathrm{eV}$, хотя измерения проводились также и в других точках спектра. В обоих случаях (монокристалл и кристалловолокно) затухание интенсивности ИКЛ на порядок величины происходит за время несколько сотен наносекунд. При больших временах затухания наблюдаются инерционные рекомбинационные процессы. Так, в случае монокристалла (рис. 5) выделен медленный компонент затухания ИКЛ, подчиняющийся гиперболической зависимости второго порядка с характерным временем около $100 \mu \mathrm{s}$ и амплитудой около $4 \%$ от начальной интенсивности.

\section{4. Обсуждение результатов}

Доминирующими излучательными переходами в оптических материалах, содержащих ионы $\mathrm{Ce}^{3+}$, являются интерконфигурационные $5 d \rightarrow 4 f$ переходы в ионе $\mathrm{Ce}^{3+}$

$$
\left(\mathrm{Ce}^{3+}\right)^{*} \rightarrow \mathrm{Ce}^{3+}+h v \text {. }
$$

При возбуждении зона-зона возможны два взаимосвязанных механизма рекомбинации зонных электронов и дырок с участием ионов церия [16]. В первом случае (2) $4 f$-электрон иона $\mathrm{Ce}^{3+}$ рекомбинирует с дыркой из валентной зоны (В3).

$$
\begin{gathered}
\mathrm{Ce}^{3+}+h^{+} \rightarrow \mathrm{Ce}^{4+} \\
\mathrm{Ce}^{4+}+e^{-} \rightarrow\left(\mathrm{Ce}^{3+}\right)^{*} .
\end{gathered}
$$

Процесс (2) является безызлучательным и может быть представлен как захват дырки из В3 на локальный уровень дырочного $\mathrm{Ce}^{3+}$-центра захвата. В результате образуется ион $\mathrm{Ce}^{4+}$, который на следующей стадии (3) может захватить электрон из зоны проводимости. Захваченный электрон релаксирует в метастабильное $5 d$-состояние, соответствующее нижнему возбужденному уровню иона $\mathrm{Ce}^{3+}$. Далее реализуется излучательный $5 d \rightarrow 4 f$-переход по схеме (1). При наличии дополнительных центров захвата электронов между стадиями (2) и (3) может быть временной промежуток, обусловленный процессом делокализации электронов с дополнительных центров захвата. Данный механизм является достаточно универсальными объясняет люминесценцию ионов $\mathrm{Ce}^{3+}$ при рекомбинации зонных носителей заряда с участием примесных ионов церия.

На рис. 6 приведена предполагаемая модель рекомбинационных процессов с участием примесных и собственных дефектов кристалла LGBO, протекающих после воздействия наносекундного пучка электронов.

В исходном состоянии LGBO : Се содержит примесные ионы церия преимущественно в зарядовом состоянии $\mathrm{Ce}^{4+}$ (состояние (a) на рис. 6). Действительно, в случае прямого фотовозбуждения LGBO : Се интенсивность фотолюминесценции примесных ионов $\mathrm{Ce}^{3+}$ невелика. Однако при возбуждении электронным пучком доминирует альтернативный механизм возбуждения, на фоне которого после окончания импульса возбуждения

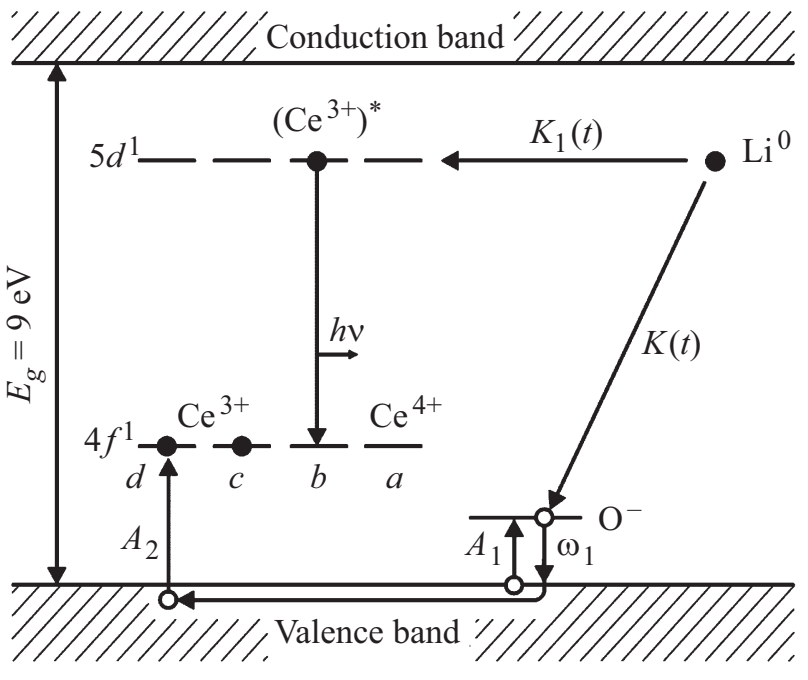

Рис. 6. Модель рекомбинационных процессов с участием примесных и собственных дефектов LGBO : Ce.

мы не видим характерного короткого интенсивного свечения $\mathrm{Ce}^{3+}$ с постоянной времени $30-40 \mathrm{~ns}$. Согласно развиваемым представлениям [9], в образце при этом происходит создание френкелевских пар дефектов типа „вакансия лития-междоузельный ион лития“", которые после перезарядки превращаются в дырочный центр $\mathrm{O}^{-}$ (дырка на $2 p$-орбитали кислорода в окрестности вакансии лития) и подвижный электронный центр $\mathrm{Li}^{0}$. Отметим, что в LGBO : Се могут присутствовать также другие типы дырочных центров, например, несколько видов $\mathrm{O}^{-}$-центров, различающихся глубиной потенциальной ямы для локализации дырки [17]. Большинство таких центров захвата являются мелкими и им соответствуют пики термостимулированной люминесценции (ТСЛ) в области $100-130 \mathrm{~K}$, но есть и центры, дающие пики ТСЛ при 250-300 K [18]. На рис. 6 показан только один тип мелкого дырочного центра с вероятностью захвата дырки из В3 $\left(A_{1}\right)$ и вероятностью термостимулированной делокализации захваченной дырки в В3 $\left(\omega_{1}\right)$.

B LGBO : Cе КОП обусловлен электронными переходами между состояниями В3 и локальным уровнем дырочного $\mathrm{O}^{-}$-центра [9]. Другой тип центра захвата представляет собой примесный ион церия. Основной $4 f^{1}$-уровень $\mathrm{Ce}^{3+}$ иона расположен в запрещенной зоне на $3.6 \mathrm{eV}$ выше потолка В3 [1]. Поэтому на нем возможен только захват дырок из В3 (2) с вероятностью $A_{2}$ (состояние $(d)$ на рис. 6). При низкой температуре все термостимулированные процессы заморожены и кинетика рекомбинационных процессов описывается системой дифференциальных уравнений

$$
\left\{\begin{array}{l}
\frac{d n_{1}}{d t}=-K(t) n_{1}(t) N \\
\frac{d n_{2}}{d t}=-K_{1}(t) n_{2}(t) N
\end{array}\right.
$$

где обозначены концентрации $\left(\mathrm{m}^{-3}\right)$ электронных $\mathrm{Li}^{0}$ центров захвата $(N)$, дырок, локализованных на мелких 
собственных $\left(n_{1}\right)$ и глубоких примесных $\left(n_{2}\right)$ центрах захвата; константы скоростей реакций $K(t)$ и $K_{1}(t)$ $\left(\mathrm{m}^{3} / \mathrm{s}\right)$, показанные на рис. 6. Здесь $K(t)$ соответствует безызлучательному туннельному переносу электрона между подвижным электронным $\left(\mathrm{Li}^{\mathrm{0}}\right)$ и дырочным $\left(\mathrm{O}^{-}\right.$центрами. Для френкелевских дефектов начальная концентрация $\mathrm{O}^{-}$центров $n_{1}(t=0)=n_{10} \approx N$, тогда „туннельный“ компонент

$$
\tilde{n}_{1}(t)=\frac{n_{10}}{1+N \int_{0}^{t} K(t) d t} .
$$

При больших временах затухания $K(t) \rightarrow$ const и асимптотическая формула для описания „хвоста“ кинетики $n_{1}(t)$ будет стремиться к гиперболе первого порядка.

Константа скорости реакции $K_{1}(t)$ соответствует туннельному переносу электрона с подвижного электронного $\mathrm{Li}^{0}$ центра на примесный дефект $\mathrm{Ce}^{4+}$. Структура этого центра на данном этапе нам не известна. Из общих соображений можно предположить, что это ион церия в позиции $\mathrm{Gd}^{3+}$, ассоциированный с каким-либо зарядокомпенсирующим дефектом, например, катионной вакансией, но может быть это ион церия в какой-либо другой кристаллографической позиции. После туннельного переноса электрона в итоге получаем возбужденный ион $\left(\mathrm{Ce}^{3+}\right)^{*}$ (состояние $(b)$ на рис. 6), который снимает возбуждение через характерную $5 d \rightarrow 4 f$-люминесценцию в области $2.8-3.3 \mathrm{eV}$. В итоге получаем ион $\mathrm{Ce}^{3+}$ в основном состоянии (состояние $(c)$ на рис. 6). Для примесных ионов церия $n_{2} \ll N$, тогда второй ,туннельный“ компонент

$$
\tilde{n}_{2}(t)=n_{20} \exp \left[-N \int_{0}^{t} K_{1}(t) d t\right]
$$

где $n_{20}-$ концентрация дырок на $\mathrm{Ce}^{4+}$ центрах в начальный момент времени.

Повышение температуры приводит к делокализации дырок с мелких центров $\left(n_{1}\right)$ и их повторному захвату на центрах $\left(n_{2}\right)$. Рассмотрим модельную систему из двух взаимодействующих дырочных центров $\left(n_{1}, n_{2}\right)$ и одного электронного центра $\left(n_{3}\right)$

$$
\left\{\begin{array}{l}
\frac{d n_{1}}{d t}=-\lambda_{1} n_{1}(t) \\
\frac{d n_{2}}{d t}=n_{1}(t) \omega_{1} \delta_{2}+n_{3}(t) \\
\frac{d n_{3}}{d t}=-\lambda_{2} n_{3}(t)
\end{array}\right.
$$

Здесь использованы обозначения $\lambda_{1}=\omega_{1}\left(1-\delta_{1}\right), \lambda_{2}-$ время жизни электронного $\mathrm{Ce}^{3+}$-центра $\left(n_{3}\right)$ в возбужденном состоянии, $\delta_{1}$ и $\delta_{2}-$ безразмерные параметры

$$
\delta_{k}=\frac{A_{k}\left(v_{k}-n_{k}\right)}{A_{1}\left(v_{1}-n_{1}\right)+A_{2}\left(v_{2}-n_{2}\right)}, \quad k=1,2 .
$$

Здесь $v_{k}-$ концентрации дефектов, служащих прекурсорами для мелких собственных $\left(v_{1}\right)$ и глубоких примесных $\left(v_{2}\right)$ дырочных центров. Решением системы уравнений (7) являются следующие зависимости для „термоактивационных“ компонентов

$$
\left\{\begin{array}{l}
n_{1}(t)=b_{10} \exp \left(-\lambda_{1} t\right) ; \\
n_{2}(t)=n_{20}+n_{10}\left[1-\exp \left(-\lambda_{1} t\right)\right]+\frac{n_{30}}{\lambda_{2}}\left[1-\exp \left(-\lambda_{2} t\right)\right] .
\end{array}\right.
$$

Величина регистрируемого сигнала $D(t)$ (КОП) с точностью до постоянного масштабного множителя соответствует концентрации $\mathrm{O}^{-}$центров $n_{1}(t)$. Аппроксимирующую функцию получим в виде суммы решений уравнений (5) и (9).

$$
D(t)=B_{1} \exp \left(-\lambda_{1} t\right)+\frac{B_{2}}{1+N \int_{0}^{t} K(t) d t} .
$$

Здесь $B_{1}$ и $B_{2}$ - параметры аппроксимации, определяющие относительный вклад каждого из процессов в регистрируемый сигнал.

Величина регистрируемого сигнала $I(t)$ (ИКЛ) с точностью до постоянного масштабного множителя соответствует $-d n_{2} / d t \propto n_{2}(t) K_{1}(t)$. Аппроксимирующую функцию получим в виде произведения решений уравнений (6) и (9).

$$
\left\{\begin{array}{l}
I(t)=\eta(t) \times I_{\text {tun }} \\
\eta(t)=1+B_{1}\left[1-\exp \left(-\lambda_{1} t\right)\right]-B_{2} \exp \left(-\lambda_{2} t\right) \\
I_{\text {tun }}=B_{0}\left(\frac{K_{1}(t)}{K_{1}(0)}\right) \exp \left[\int_{0}^{t} K_{1}(t) d t\right]
\end{array}\right.
$$

Здесь $\eta(t)$ - функция выхода, учитывающая интерактивный компонент; $B_{0}-$ масштабный множитель; $B_{1}$ и $B_{2}$ - параметры аппроксимации, определяющие относительный вклад интерактивных процессов в регистрируемый сигнал; $I_{\text {tun }}(t)$ - интенсивность туннельной

Параметры модели LGBO при $293 \mathrm{~K}$, описывающие кинетику КОП при $1.8 \mathrm{eV}$ и кинетику ИКЛ при $3.0 \mathrm{eV}$

\begin{tabular}{l|c|c|c|c}
\hline \multirow{2}{*}{ Параметр } & \multicolumn{2}{|c|}{ КОП } & \multicolumn{2}{c}{ ИКЛ } \\
\cline { 2 - 5 } & $(\mathrm{CF})$ & $(\mathrm{CF})$ & $(\mathrm{CF})$ & $(\mathrm{SC})$ \\
\hline $\mathrm{B}_{0}$, arb.units & - & - & 1.630 & 1.116 \\
$\mathrm{~B}_{1}$, arb.units & 0.295 & - & 0.001 & 1.985 \\
$\lambda_{1}^{-1}$, ns & 87.3 & - & 87.0 & 2600 \\
$\mathrm{~B}_{2}$, arb.units & 0.125 & 0.373 & 0.699 & 0.463 \\
$\lambda_{2}^{-1}, \mathrm{~ns}$ & - & - & 32.2 & 27.1 \\
$N, 10^{23} \mathrm{~m}^{-3}$ & 7.00 & 14.5 & 7.4 & 5.8 \\
\hline FOM,\% & 4.97 & $<1.00$ & 4.77 & $7.35^{*}$
\end{tabular}

Примечание. * FOM для кристалловолокна (CF) и монокристалла (SC) рассчитан для одного и того же диапазона времен затухания $10 \mathrm{~ns}-5 \mu \mathrm{s}$. Прочерк означает, что данный параметр не используется. 
люминесценции. Качество аппроксимации оценивали с использованием стандартного критерия FOM (Figure Of Merit)

$$
\mathrm{FOM}=\frac{\sum_{i}\left|y_{i}-y\left(t_{i}\right)\right|}{\sum_{i} y_{i}},
$$

где $t_{i}$ - моменты времени затухания, для которых получены экспериментальные данные $y_{i}$ и рассчитаны теоретические значения $y\left(t_{i}\right)$. Результаты наилучшей аппроксимации представлены в таблице и на рис. 2, 4 и 5.

\section{5. Заключение}

Спектры и кинетика ИКЛ и КОП монокристаллов и кристалловолоконных образцов LGBO : Се экспериментально исследованы при возбуждении электронным пучком наносекундной длительности. Экспериментальные данные сопоставлены с результатами численного моделирования рекомбинационных процессов. На основании полученных экспериментальных и расчетных данных сделаны обоснованные заключения относительно происхождения интенсивной ИКЛ LGBO : Cе, спектр которой сосредоточен в $5 d-4 f$-полосе люминесценции $\mathrm{Ce}^{3+}(2.5-3.4 \mathrm{eV})$.

Согласно предложенному механизму возбуждения ИКЛ, при воздействии электронного пучка в окружении четырехвалентных ионов церия формируются короткоживущие электронные $\mathrm{Li}^{0}$-центры. Туннельный перенос электрона между $\mathrm{Li}^{0}$ и $\mathrm{Ce}^{4+}$-центрами приводит к перезарядке примесного иона и формированию короткоживущего примесного $\mathrm{Ce}^{3+}$-центра в возбужденном состоянии. Снятие этого возбуждения приводит к дополнительному вкладу в люминесценцию в полосе свечения $\mathrm{Ce}^{3+}$. Кинетика затухания ИКЛ в рамках данного механизма контролируется реакцией туннельного переноса электрона в паре $\left\{\mathrm{Li}^{0}, \mathrm{Ce}^{4+}\right\}$. Постоянная времени такой реакции заметно превышает радиационное время жизни возбужденного иона $\mathrm{Ce}^{3+}$. Это обусловливает отличия кинетики затухания ИКЛ от кинетики затухания фотолюминесценции $\mathrm{Ce}^{3+}$. Дополнительные особенности кинетики затухания ИКЛ обусловлены взаимодействием мелких дырочных центров, являющихся источником делокализованных дырок, и глубокими дырочными центрами в виде примесных ионов церия.

Авторы признательны Л.И. Исаенко и И.Н. Седуновой за предоставление образцов боратов лития для исследования, Д.О. Вострову за помощь в проведении экспериментов.

\section{Список литературы}

[1] I.N. Ogorodnikov, V.A. Pustovarov. J. Lumin. 134, 113 (2013).

[2] I.N. Ogorodnikov, V.A. Pustovarov, S.I. Omelkov, D.O. Vostrov, L.I. Isaenko. Opt. Mater. 36, 6, 1060 (2014).

[3] J.-P. Chaminade, O. Viraphong, F. Guillen, C. Fouassier, B. Czirr. IEEE Trans. Nucl. Sci. 48, 4, 1158 (2001).
[4] C.W.E. van Eijk. Rad. Meas. 38, 4-6, 337 (2004).

[5] И.Н. Огородников, Н.Е. Порывай, В.А. Пустоваров, А.В. Толмачев, Р.П. Явецкий, В.Ю. Яковлев. ФТТ 51, 6 , 1097 (2009).

[6] I.N. Ogorodnikov, N.E. Poryvay, V.A. Pustovarov, A.V. Tolmachev, R.P. Yavetskiy, V.Yu. Yakovlev. Rad. Meas. 45, 3-6, 336 (2010).

[7] F. Yang, S.K. Pan, D.Z. Ding, G.H. Ren. Cryst. Res. Tech. 44,2, 141 (2009).

[8] Р.П. Явецкий, А.В. Толмачев. Письма ЖТФ 30, 23, 8 (2004).

[9] И.Н. Огородников, М.С. Киселева, И.Н. Седунова. ХВЭ 46, 3, 199 (2012).

[10] И.Н. Огородников, М.С. Киселева. ЖЭТФ 142, 1, 169 (2012).

[11] И.Н. Огородников, И.Н. Седунова, В.Ю. Иванов, Л.И. Исаенко. ФТТ 54,10, 1914 (2012).

[12] T.S. Koroleva, M.M. Kidibaev, A. Nehari, Ch. Pedrini, K. Lebbou, A.N. Belsky, A.N. Tcherepanov, A.V. Ishchenko, V.Yu. Ivanov, I.N. Sedunova, O.S. Teslenko, L.V. Viktorov, B.V. Shulgin, L.H. Zheng, J. Xu, V. Kononets, O. Sidletskiy. Opt. Mater. 35, 5, 868 (2013).

[13] И.Н. Огородников, И.Н. Седунова, В.Ю. Иванов, Б.В. Шульгин, А.В. Ищенко, М.М. Кидибаев, Т.С. Королева. Оптика и спектроскокпия, 115, 1, 68 (2013).

[14] В.Ю. Яковлев. ФТТ 34, 4, 1231 (1992).

[15] В.М. Лисицын, В.И. Корепанов. Спектральные измерения с временным разрешением. Изд. ТПИ, Томск (2008), 90 с.

[16] W.W. Moses, S.E. Derenzo, M.J. Weber, A.K. Ray-Chaudhuri, F. Cerrina. J. Lumin. 59, 89 (1994).

[17] И.Н. Огородников, М.С. Киселева. ФТТ 54, 4, 697 (2012).

[18] I.N. Ogorodnikov, D.O. Vostrov, V.A. Pustovarov, I.N. Sedunova. Opt. Mater. 36, 9, 1571 (2014).

Редактор К.В. Емцев 